○ MICHEL FRIEDRICH, DOMINIQUE FRIEDRICH, ARNE TERJUNG, GUNTHER ROGMANS, THOMAS FRIELING, CLAYTON KRAFT, BERNHARD HEMMERLEIN, ALEXANDER KHUDYAKOV

UDK 618.11-006.6-033.2-021.3-06:618.2

DOI: $10.20333 / 25000136-2021-5-67-70$

\title{
Casuistics of primary metastatic ovarian cancer in pregnancy
}

Michel Friedrich ${ }^{1}$, Dominique Friedrich ${ }^{1}$, Arne Terjung ${ }^{1}$, Gunther Rogmans ${ }^{1}$, Thomas Frieling ${ }^{2}$, Clayton Kraft ${ }^{3}$, Bernhard Hemmerlein ${ }^{4}$, Alexander Khudyakov ${ }^{1}$

${ }^{1}$ Klinik für Frauenheilkunde und Geburtshilfe, Helios Klinikum, Lutherplatz 40, D-47805 Krefeld, Germany

${ }^{2}$ Klinik für Gastroenterologie, Hepatologie und Onkologie, Helios Klinikum, Lutherplatz 40, D-47805 Krefeld, Germany

${ }^{3}$ Klinik für Orthopädie und Unfallchirurgie, Helios Klinikum, Lutherplatz 40, D-47805 Krefeld, Germany

${ }^{4}$ Institut für Pathologie, Helios Klinikum, Lutherplatz 40, D-47805 Krefeld, Germany

Abstract. Malignancies during pregnancy are usually treated similarly to the malignancies in non-pregnant patients. Results of controlled therapeutic trials are typically not available. However, registered studies associated with some of the most common malignancies exist. In case of ovarian cancer, due to its rarity - especially with the primary metastatic cases - only individual case reports are available. The article presents a case with par aortal abdominal, thoracic and cervical lymph node metastasis in a 33-years-old pregnant female. She was treated with primary systemic chemotherapy since early pregnancy. At 32 weeks of gestation, the patient gave birth to a girl $(1460 \mathrm{~g})$ by caesarean section. Subsequently, the patient received 3 more cycles of chemotherapy with carboplatin/ paclitaxel and bevacizumab for 30 months as well as letrozole maintenance therapy. Three years after the initial diagnosis, complete instrumental assessment showed unremarkable results without tumour recurrence.

Key words: primary ovarian cancer, pregnancy, chemotherapy, surgery.

Conflict of interest. The authors declare the absence of obvious and potential conflicts of interest associated with the publication of this article.

Citation: Michel Friedrich, Dominique Friedrich, Arne Terjung, Gunther Rogmans, Thomas Frieling, Clayton Kraft, Bernhard Hemmerlein, Alexander Khudyakov. Casuistics of primary metastatic ovarian cancer in pregnancy. Siberian Medical Review. 2021;(5):67-70. DOI: 10.20333/25000136-2021-5-67-70

\section{Introduction}

Cancer diseases in pregnancy have an incidence of 1:1000. These are primarily malignant melanoma (2.6:1000), Hodgkin's lymphoma (1:1,000-6,000), breast cancer $(1: 3,000-10,000)$ and cervical carcinoma $(1 \cdot 2: 10,000)$. Ovarian carcinoma only occurs in approximately $1-2$ cases out of 100,000 pregnancies $[1,2]$. Based on German statistics, this affects about 6-12 patients per year, making ovarian cancer one of the least severe malignancies.

Malignancies during pregnancy are usually treated similarly to the respective malignancies in non-pregnant patients. Results of controlled therapeutic trials are usually unavailable. However, register studies associated with some of the most common malignancies exist. In case of ovarian cancer, however, due to its rarity especially of primary metastatic cases - only individual case reports are available.

\section{Case report}

A 33-years-old patient was present at the emergency department at 11 weeks of gestation with swelling in her left arm. Phlebothrombosis of the left subclavian vein was suspected. Duplex sonography was performed showing pronounced cervical lymphomas in addition to free perfusion of all vessels. Histological assessment revealed presence of cervical lymph node metastases of estrogen receptor-positive adenocarcinoma.
As part of the tumour search, the gynaecological examination revealed cystic space-consuming lesions in recto-uterine cavity as well as a timely and unremarkable pregnancy.

Histological confirmation of low-grade serous ovarian cancer was performed during laparoscopic adnexectomy based on a borderline tumour of the right ovary. MRI staging of the chest and abdomen revealed the presence of stage cT3a, cN1, pM1, FIGO IVB with paraaortal abdominal, thoracic and cervical lymph node metastases.

After a detailed explanation and presentation of the second and third opinions, the patient wished to maintain the pregnancy and to undergo primary systemic chemotherapy.

Four cycles of carboplatin/pacliaxel (AUC5/175mg/ $\mathrm{m}^{2} \mathrm{KOF}, \mathrm{q} 3 \mathrm{w}$ ) showed a favourable response of cervical lymph node metastases and a decrease of tumour marker Ca 12-5. Due to inconspicuous IUGR of the fetus, the therapy with carboplatin mono (AUC5, q3w) was cancelled. This led to the onset of progressive neurological symptoms with oscillopsia. Upon ruling out of preeclampsia, the fetal lung maturation was performed through administering $2 \times 12 \mathrm{mg}$ of betametasone i.m. at 28 weeks of gestation. After the second administration of carboplatin mono increasing headache and vision disorders were experienced at 31 weeks of gestation, an MRI of the skull showed numerous changes interpreted 
as paraneoplastic cerebral infarctions.

Against the background of effective anticoagulation, progression of neurological symptoms and manifestations of preeclampsia occurred. At 32 weeks of gestation, the patient gave birth to a girl $(1460 \mathrm{~g})$ through caesarean section. The newborn did not have any complications except for persistent ductus arteriosus Botalli.

Postpartum staging showed complete regression of lymphadenopathy. Three weeks later, modified posterior longitudinal exenteration with hysterectomy, left adnexectomy, sigma resection, pelvis peritonectomy, diaphragm domes and paracolic, appendectomy, infragastric omentectomy, pelvinian and paraaortal lymphonodectomy as well as HIPEC with $50 \mathrm{mg} / \mathrm{m}^{2}$ cisplatin was performed.

Post-operative skull MRI showed complete regression of parenchyma findings, which were retrospectively qualified as posterior reversible encephalopathy syndrome (PRES).

The patient subsequently received 3 more cycles of chemotherapy with carboplatin/paclitaxel and bevacizumab for 30 months as well as letrozole maintenance therapy.

Three years after the initial diagnosis, complete instrumental assessment was carried out showing unremarkable results without tumour recurrence. Tumour marker Ca 12-5 was in the normal range.

\section{Discussion}

Similarly to treatment of other malignancies in pregnancy, the therapy strategy depends on the gestational age and the degree of the pathologic process progression. Although no therapy options that would improve prognosis should be withheld from pregnant patients, it is important to include the prognosis for the fetus in the therapy concept. Generally, both surgical therapy and cytostatic chemotherapy are feasible.

In case of the diagnosis established during the first trimester, it must be discussed in consensus with the mother whether pregnancy continuation or therapeutic abortion for delivery of stage-appropriate therapy is desired. Infertility caused by the therapy must be taken into consideration.

If sufficient cytoreduction appears achievable without hysterectomy, adequate surgery is also possible with continuation of pregnancy. In the first trimester, transitory progesterone replacement is necessary before 10 weeks of gestation after adnexectomy on both sides. Starting from the second trimester, placental secretion of steroid hormones is decisive and sufficient for the maintenance of pregnancy [3].

However, the perioperative risk is to be regarded as increased due to the known pregnancy-typical changes such as increased cardiac output, increased oxygen demand, thrombotic tendency and increased perfusion of genitals. In addition, from the second trimester onwards, the altered anatomical conditions challenge the technical feasibility of the surgery.

If sufficient cytoreduction is impossible or associated with high risk, neoadjuvant chemotherapy may be carried out until parturition, in line with the recommendations for non-pregnant patients [4].

All cytostatic agents are considered as substances of risk category D during pregnancy. There is limited clinical experience in treatment of mammary carcinomas and hematologic malignancies, where the risk of malformations and complications depends on the substance and the duration of exposure: while the risk of malformations in the first trimester with alkylating agents is approximately $16 \%$, it decreases to around $8 \%$ in the second trimester [5]. Such substances as carboplatin and paclitaxel administered as first-line therapy against ovarian cancer become involved from the 2nd trimester and may be used with reasonable risk. The following complications are associated with the use of carboplatin: IUGR, premature birth, oligo - and polyhydramnions. To the date, these malformations have not been observed during the second and the third trimesters [6]. Experience with Taxanen is also limited in published literature, but its use also seems to be associated with a rather low risk [7]. Administration of bevacizumab, on the other hand, is completely unexplored and seems to be contraindicated based on theoretical considerations.

Regarding the timing of therapy and parturition, it should be noted that if chemotherapy is administered within 3 weeks before parturition, increased complication rates due to fetal and maternal myelosuppression are to be expected, whereas administration immediately before birth leads to increased complication rates for the newborn due to interrupted placental and severely restricted hepatic and renal elimination.

Premedication for chemotherapy should be based on long-established drugs such as MCP, dimenhydrinate or Meclozin. In contrast to NK-1 inhibitors, there is at least limited experience with the use of odansetron during pregnancy. In the case of steroids, physiological glucocorticoids such as prednisolone are preferable, as $>90 \%$ of them are inactivated by placental enzyme system, while 9- $\alpha$-fluorinated glucocorticoids (dexamethasone, betamethasone) lead to significantly higher fetal plasma levels. 


\section{Ca $12-5(\mathrm{U} / \mathrm{mI})$}

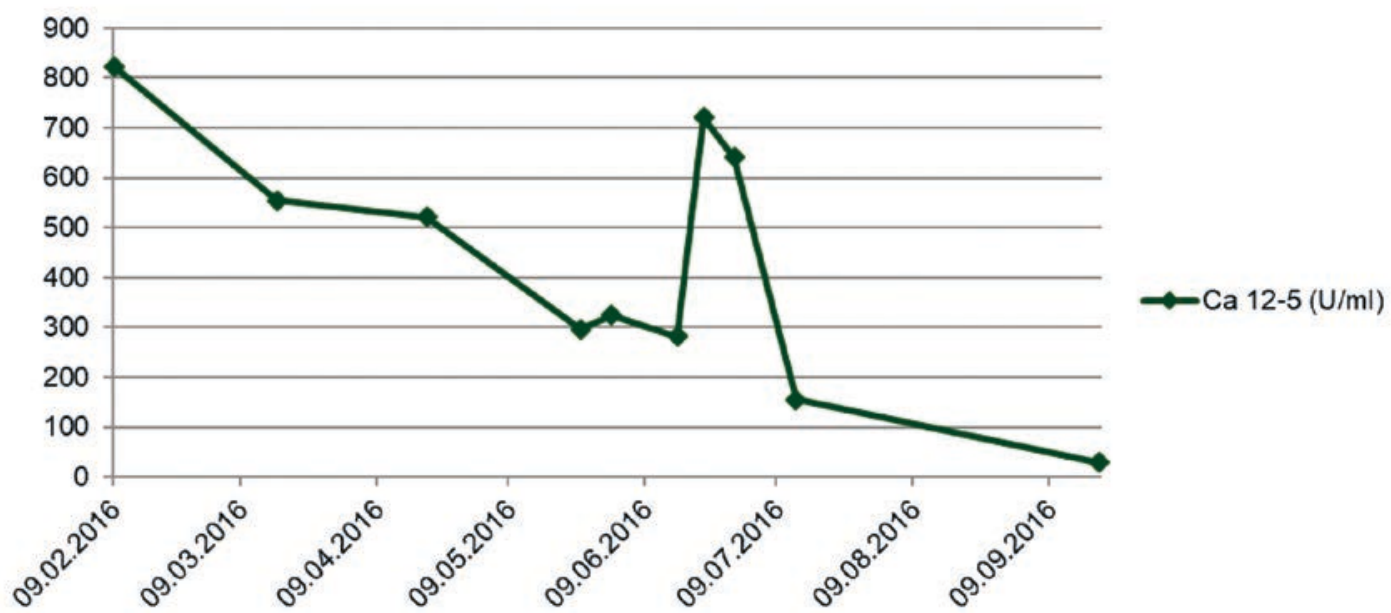

Figure 1. Change in the serum level of tumor marker Ca 12-5.

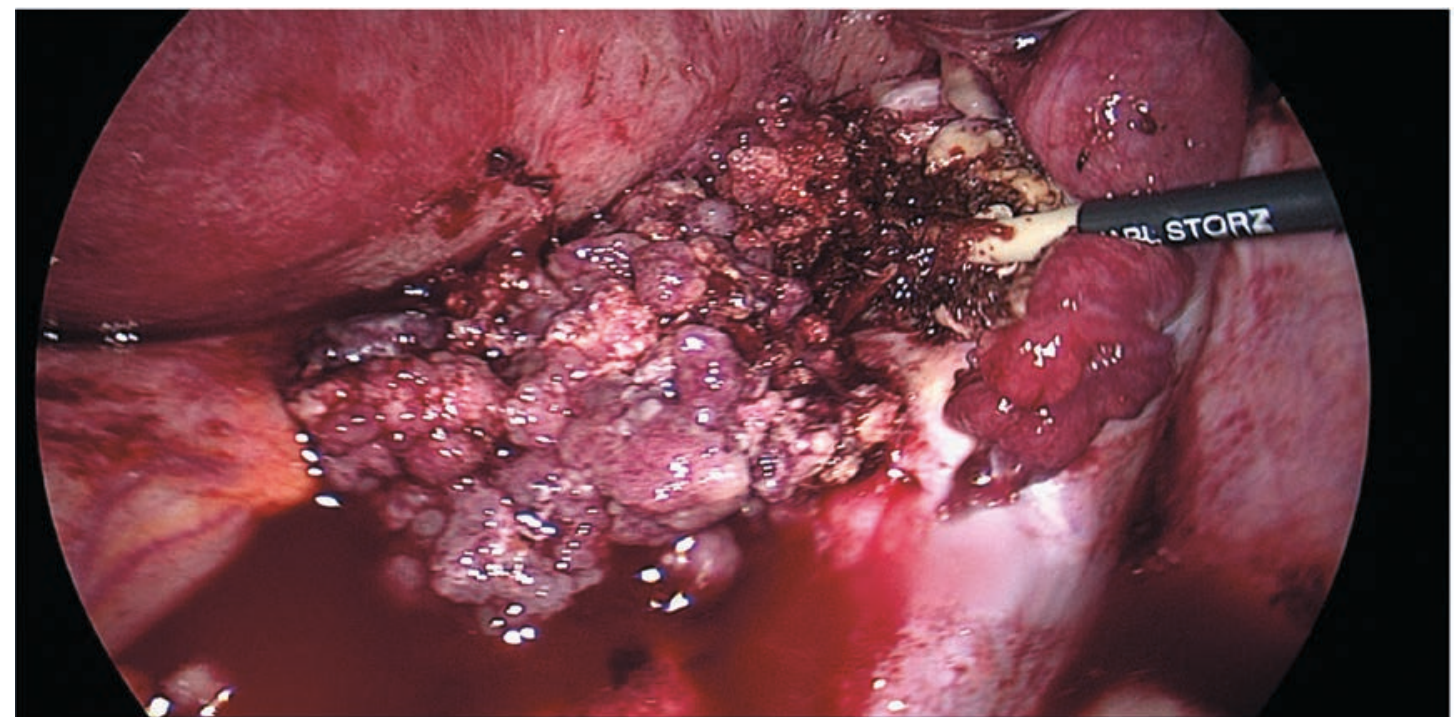

Figure 2. Laparoscopic image of the ovarian tumour on the right while establishing the diagnosis.

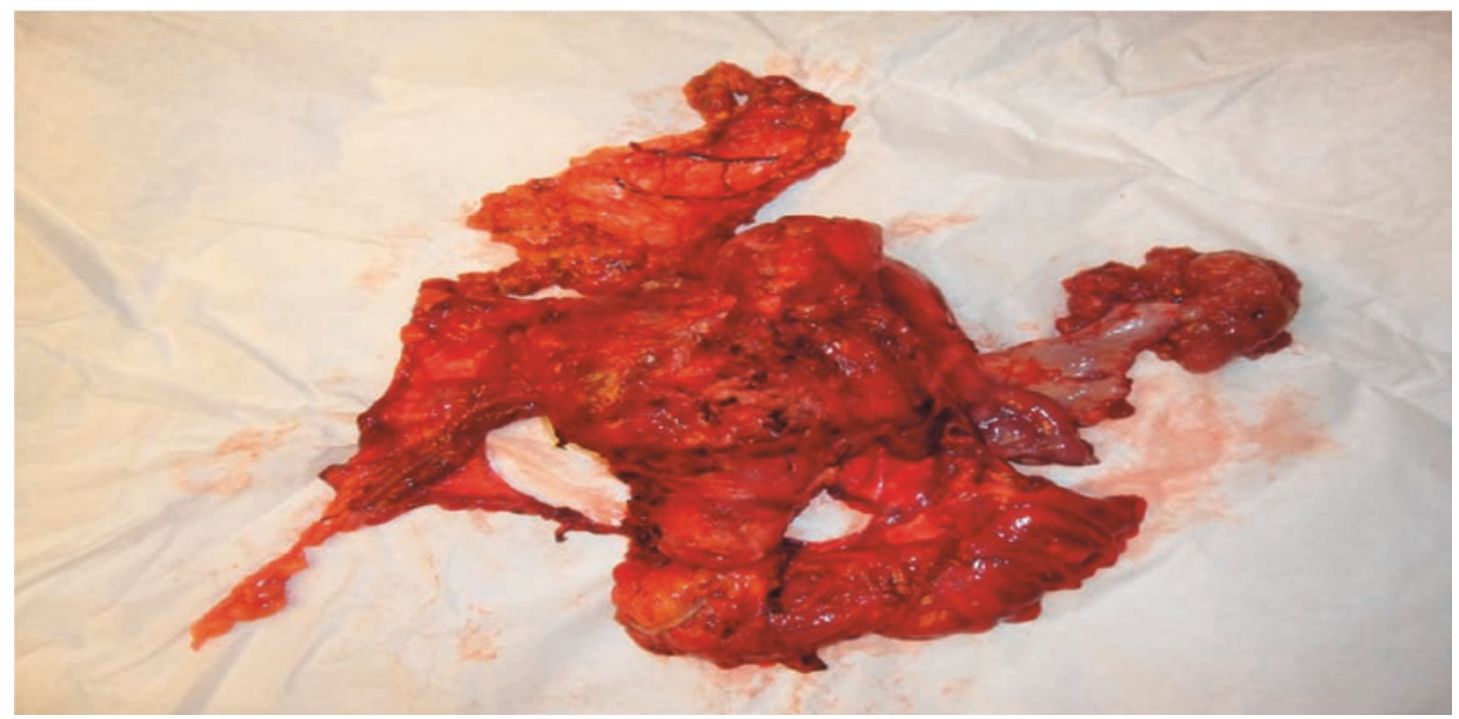

Figure 3. Operative material with the uterus, left adnexa, sigma and peritoneum of the lesser pelvis. 


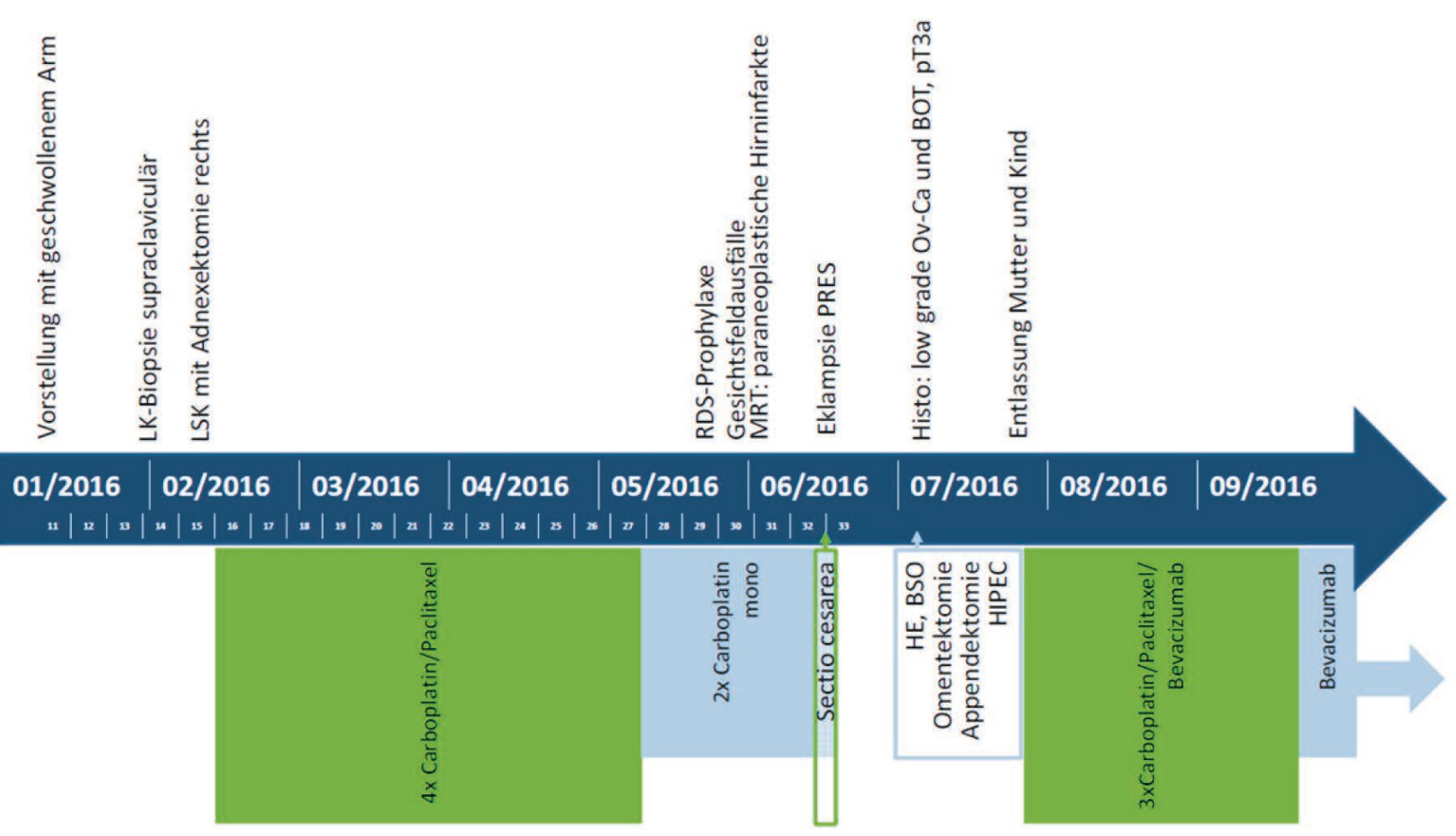

Figure 5. Timing of diagnostics and therapy.

\section{References}

1. Pavlidis NA. Coexistence of Pregnancy and Malignancy. The Oncologist. 2002; (7): 279-287.

2. Zhao XY, Huang HF, Lian LJ, Lung JH. Ovarian cancer in pregnancy: a clinicopathologic analysis of 22 cases and review of the literature. International Journal of Gynecological Cancer. 2006; 16(1): 8-15.

3. Csapo AI, Pulkkinen MO, Ruttner B, Sauvage JP, Wiest WG. The significance of the human corpus luteum in pregnancy maintenance. I. Preliminary studies. American Journal of Obstetrics and Gynecology. 1972; 112(8): 1061-7.

4. Wright AA, Bohlke K, Armstrong DK, Bookman MA, Cliby WA, Coleman RL, Dizon DS, Kash JJ, Meyer L A, Moore KN, Olawaiye AB, Oldham J, Salani R, Sparacio D, Tew W P, Vergote I, Edelson MI . Neoadjuvant chemotherapy for newly diagnosed, advanced ovarian cancer: Society of Gynecologic Oncology and American Society of Clinical Oncology Clinical Practice Guideline. Gynecologic Oncology. 2016;143(1):3-15. DOI: 10.1016/j.ygyno.2016.05.022

5. Selig BP, Furr JR, Huey RW, Moran C, Alluri VN, Medders GR, Mumm C D, Hallford HG, Mulvihill JJ. Cancer chemotherapeutic agents as human teratogens. Birth Defects Research. Part A, Clinical and Molecular Teratology. 2012; 94(8): 626-50. DOI: 10.1002/bdra.23063. Epub 2012 Jul 31
6. Mir O, Berveiller P, Ropert S, Goffinet F, Goldwasser F. Use of platinum derivatives during pregnancy. Cancer. 2008; 113(11): 3069.

7. Zagouri F, Sergentanis TN, Chrysikos D, Filipits M, Bartsch R. Taxanes for ovarian cancer during pregnancy: a systematic review. Oncology. 2012; 83(4): 234.

8. Runowicz CD, Brewer M. Chemotherapy of ovarian cancer in pregnancy (Review); 2019 UpToDate; www. uptodate.com @2019 UpToDate, Inc. and/or its affiliates. All Rights Reserved

\section{Author information}

Michael Friedrich, Prof. Dr. Med, Head Physician of the Department of Gynecology and Obstetrics, Helios Hospital, (Krefeld, Germany); Phone: 0049(0)2151-322201; e-mail: michael. friedrich@helios-gesundheit.de

Dominique Friedrich, Gynecology and Obstetrics, Helios Hospital, (Krefeld, Germany); e-mail:michael.friedrich@helios-gesundheit.de

Arne Terjung, Gynecology and Obstetrics, Helios Hospital (Krefeld, Germany); Phone: 0049 (21 51)32-22 01; e-mail: euregio-tumorboard@helios-kliniken.de

Gunther Rogmans, Gynecology and Obstetrics, Helios Hospital, (Krefeld, Germany); e-mail: gynaekologie@klinikum-lev.de.

Thomas Frieling, Clinic for Gastroenterology, Hepatology and Oncology, Helios Clinic. e-mail: thomas.frieling@helios-gesundheit.de

Clayton Kraft, Clinic for Orthopedics and Trauma Surgery, Helios Clinic; e-mail: clayton. kraft@helios-gesundheit.de

Bernhard Hemmerlein, Institute for Pathology, Helios Clinic; e-mail: bernhard. hemmerlein@helios-gesundheit.de

Alexander Khudyakov, Gynecology and Obstetrics, Helios Hospital; e-mail: info@gtkkrefeld.de

Received 21 September 2021 Revision Received 24 September 2021 Accepted 29 September 2021 